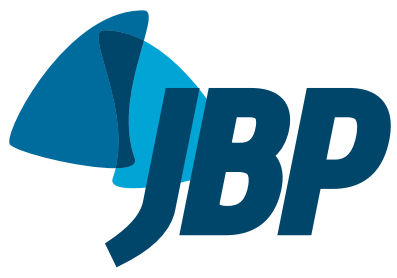

\title{
Endobronchial ultrasound in esophageal cancer - when upper gastrointestinal endoscopy is not enough
}

\author{
Lília Maia Santos ${ }^{1,2, a}$, Márcia Jacomelli1,b , Paulo Rogério Scordamaglio ${ }^{1, c}$,
} Paulo Francisco Guerreiro Cardoso ${ }^{3, \mathrm{~d}}$, Viviane Rossi Figueiredo,

\section{TO THE EDITOR:}

Endobronchial ultrasound-guided transbronchial needle aspiration (EBUS-TBNA) and endoscopic ultrasoundguided fine-needle aspiration (EUS-FNA) are minimally invasive, safe, and accurate techniques for sampling hilar and mediastinal lymph nodes and masses. ${ }^{(1)}$ They are considered to be complementary techniques, and the combination of the two can reach virtually all mediastinal nodal stations, being highly accurate for lung and esophageal cancer staging. ${ }^{(1,2)}$

Esophageal cancer is the sixth leading cause of cancerrelated death worldwide, and upper gastrointestinal endoscopy (UGIE) is the gold standard for its diagnosis. ${ }^{(3)}$ Although EUS-FNA is primarily used for locoregional staging of esophageal cancer, being the gold standard method for this purpose, it can be used for diagnosing esophageal cancer in cases in which there is a high suspicion of esophageal malignancy and UGIE does not provide a definitive diagnosis. ${ }^{(2-4)}$ Because EUS-FNA and UGIE are both performed through the esophagus, they can be technically challenging in the presence of a malignant stricture that obliterates the esophageal lumen. Given the proximity between the esophagus and the tracheobronchial tree, EBUS-TBNA can be an alternative diagnostic technique in such cases. Here, we report a case in which EBUS-TBNA was used for diagnosing esophageal carcinoma.

A 60-year-old woman presented with a one monthhistory of odynophagia, progressive dysphagia, and weight loss (of $10 \mathrm{~kg}$ ). A CT scan of the neck and chest showed nonspecific thickening and mild heterogeneous enhancement at the pharyngoesophageal junction (Figure $1 \mathrm{~A})$. UGIE showed a vegetative lesion that partially obstructed the esophageal lumen and prevented the progression of the endoscope. A biopsy of the lesion showed low-grade squamous epithelial dysplasia. Flexible bronchoscopy showed no endobronchial lesions or signs of extrinsic compression. A positron emission tomography scan revealed circumferential thickening of the cervical esophagus leading to narrowing of the esophageal lumen (standardized uptake value, 9.6), as well as nonspecific subcentimetric mediastinal lymph nodes (subcarinal lymph node standardized uptake value, 2.2), together with no signs of invasion into the adjacent structures (Figure 1B).
Because of the proximity of the lesion to the upper esophageal sphincter and because esophageal stenosis prevented the passage of the endoscope for a second biopsy, an EBUS-TBNA was performed; EBUS showed a heterogeneous lesion with well-defined margins and no vessels at the subglottic level (Figure $1 \mathrm{C}$ ), and 22-gauge TBNA findings were consistent with squamous cell carcinoma of the esophagus.

The clinical hallmarks of esophageal cancer are progressive dysphagia and weight loss. ${ }^{(3,4)}$ However, patients with esophageal cancer often present with few or no symptoms; dysphagia occurs only when two thirds of the esophageal lumen are involved, which is why diagnosis is often delayed and prognosis is poor. ${ }^{(3-5)}$

Esophageal cancer can present as a flat, subtle area or as a lumen-obscuring mass. ${ }^{(3)}$ UGIE allows detailed tumor analysis and tissue sampling. ${ }^{(3,4)}$ At least seven biopsies should be performed in the suspected area for adequate sampling, brushings alone being inadequate. ${ }^{(3,4)}$ When UGIE fails to provide a definitive diagnosis, EUS-FNA should be considered. (4) However, EUS-FNA probes are typically large and can therefore be a problem in the presence of a malignant stricture. ${ }^{(4)}$ In addition, although local extension to lymph nodes and esophageal wall invasion are best investigated by EUS-FNA, ${ }^{(2,4)}$ narrowing of the esophageal lumen can prevent the progression of the endoscope in up to $30 \%$ of cases, ${ }^{(5)}$ constituting an obstacle to diagnosis and staging by preventing the collection of representative biopsy material through the esophageal lumen. In such cases, EBUS-TBNA can be performed either through the esophagus (because EBUS-TBNA probes are smaller in diameter) or through the tracheobronchial tree, constituting an alternative approach. ${ }^{(2,5)}$

EBUS-TBNA is indicated for the diagnosis of mediastinal lesions that are adjacent to or in direct contact with the tracheobronchial tree, allowing visualization of mediastinal structures such as lymph nodes, vessels, and the esophagus itself. ${ }^{(1)}$ In addition to mediastinal staging of lung cancer, EBUS-TBNA has been described for esophageal cancer staging and as a diagnostic method for centrally located lung cancer and benign/malignant mediastinal and hilar lymphadenopathy. ${ }^{(1,2)}$

1. Serviço de Endoscopia Respiratória, Disciplina de Pneumologia, Instituto do Coração - InCor - Hospital das Clínicas, Faculdade de Medicina, Universidade de São Paulo, São Paulo (SP) Brasil.

2. Serviço de Pneumologia, Hospitais da Universidade de Coimbra, Centro Hospitalar e Universitário de Coimbra, Coimbra, Portugal.

3. Departamento de Cardiopneumologia, Disciplina de Cirurgia Torácica, Instituto do Coração - InCor - Hospital das Clínicas, Faculdade de Medicina,

Universidade de São Paulo, São Paulo (SP) Brasil

a. (iD) http://orcid.org/0000-0003-3215-4701; b. (iD) http://orcid.org/0000-0001-8657-458X; c. (iD) http://orcid.org/0000-0001-8971-5333;

d. (iD http://orcid.org/0000-0003-0749-4165; e. (iD http://orcid.org/0000-0002-9458-8153 
Garrido et al. described the use of EBUS alone (i.e., without biopsy) for assessing tracheobronchial invasion in patients with malignant esophageal stricture. ${ }^{(5)}$ EBUS has been shown to be highly specific and sensitive for detecting tracheobronchial invasion, because it allows high-resolution evaluation of the outermost layer of the tracheobronchial tree. ${ }^{(4)}$ The use of EBUS-TBNA in combination with EUS-FNA has been shown to improve the staging of esophageal cancer by sampling of lymph nodes that are poorly assessed or cannot be assessed by EUS-FNA, including peritumoral lymph nodes. ${ }^{(1,2)}$ EBUS-TBNA contributes to esophageal cancer staging by allowing evaluation of right paratracheal lymph nodes-which are often difficult to visualize with EUS-FNA because of air interposition from the trachea-and hilar lymph nodes that cannot be reached by EUS-FNA. ${ }^{(1,2)}$ When the peritumoral lymph nodes are sampled by EUS-FNA, there is a risk of specimen contamination secondary to piercing the primary tumor; this can be avoided with EBUS-TBNA performed through the tracheobronchial tree. ${ }^{(2)}$

To our knowledge, there have been no data on the usefulness of EBUS-TBNA for the diagnosis of esophageal cancer by means of sampling performed through the tracheobronchial tree. Our study adds to the literature by reporting on a patient in whom malignant esophageal stricture prevented the collection of representative biopsy material through the esophageal lumen, a definitive diagnosis of primary esophageal cancer being made by EBUS-TBNA performed through the tracheobronchial tree. Given the proximity between the tracheobronchial tree and the esophagus, collaboration between the gastrointestinal endoscopist and the bronchoscopist is intuitive and desired in order to improve diagnostic accuracy.
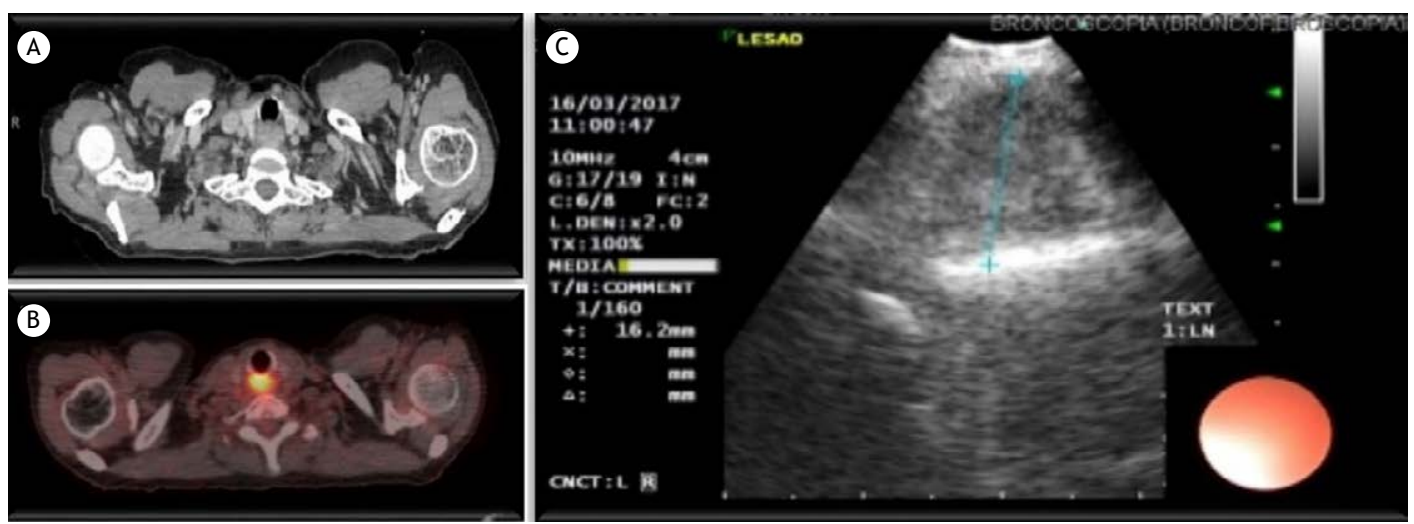

Figure 1. In $A, C T$ scan of the neck and chest showing nonspecific thickening and mild heterogeneous enhancement at the pharyngoesophageal junction. In B, positron emission tomography scan showing circumferential thickening of the cervical esophagus leading to narrowing of the esophageal lumen (standardized uptake value, 9.6). Note the absence of signs of invasion into the adjacent structures. In C, endobronchial ultrasound showing a heterogeneous lesion with well-defined margins and no vessels at the subglottic level, where endobronchial ultrasound-guided transtracheal needle aspiration was performed.

\section{REFERENCES}

1. Vilmann $P$, Frost Clementsen $P$, Colella $S$, Siemsen $M$, De leyn $P$, Dumonceau JM, et al. Combined endobronchial and esophageal endosonography for the diagnosis and staging of lung cancer: European Society of Gastrointestinal Endoscopy (ESGE) Guideline, in cooperation with the European Respiratory Society (ERS) and the European Society of Thoracic Surgeons (ESTS). Eur J Cardiothorac Surg. 2015;48(1):1-15. https://doi.org/10.1093/ejcts/ezv194

2. Liberman $M$, Hanna $N$, Duranceau $A$, Thiffault $V$, Ferraro P. Endobronchial ultrasonography added to endoscopic ultrasonography improves staging in esophageal cancer. Ann Thorac Surg. 2013;96(1):232-6: discussion 236-8. https://doi.org/10.1016/j. athoracsur.2013.03.023
3. Alsop BR, Sharma P. Esophageal Cancer. Gastroenterol Clin North Am. 2016;45(3):399-412. https://doi.org/10.1016//.gtc.2016.04.001

4. Varghese TK Jr, Hofstetter WL, Rizk NP, Low DE, Darling GE, Watson $T J$, et al. The society of thoracic surgeons guidelines on the diagnosis and staging of patients with esophageal cancer. Ann Thorac Surg. 2013;96(1):346-56. https://doi.org/10.1016/..athoracsur.2013.02.069

5. Garrido T, Maluf-Filho F, Sallum RA, Figueiredo VR, Jacomell M, Tedde M. Endobronchial ultrasound application for diagnosis of tracheobronchial tree invasion by esophageal cancer. Clinics (Sao Paulo). 2009;64(6):499-504. https://doi.org/10.1590/S180759322009000600003 